\title{
Front Matter: Volume 11073
}

, "Front Matter: Volume 11073," Proc. SPIE 11073, Clinical and Preclinical Optical Diagnostics II, 1107301 (20 September 2019); doi: 10.1117/12.2542841

SPIE. Event: European Conferences on Biomedical Optics, 2019, Munich, Germany 


\title{
Clinical and Preclinical Optical Diagnostics II
}

\author{
J. Quincy Brown \\ Ton G. van Leeuwen \\ Editors
}

\section{3-25 June 2019 \\ Munich, Germany}

Sponsored by

The Optical Society (United States)

SPIE

Published by

SPIE 
The papers in this volume were part of the technical conference cited on the cover and title page. Papers were selected and subject to review by the editors and conference program committee. Some conference presentations may not be available for publication. Additional papers and presentation recordings may be available online in the SPIE Digital Library at SPIEDigitallibrary.org.

The papers reflect the work and thoughts of the authors and are published herein as submitted. The publisher is not responsible for the validity of the information or for any outcomes resulting from reliance thereon.

Please use the following format to cite material from these proceedings:

Author(s), "Title of Paper," in Clinical and Preclinical Optical Diagnostics II, edited by J. Quincy Brown, Ton G. van Leeuwen, Proceedings of SPIE-OSA Vol. 11073 (SPIE, Bellingham, WA, 2019) Sevendigit Article CID Number.

ISSN: 1605-7422

ISSN: $2410-9045$ (electronic)

ISBN: 9781510628397

ISBN: 9781510628403 (electronic)

Copublished by

SPIE

P.O. Box 10, Bellingham, Washington $98227-0010$ USA

Telephone +1 3606763290 (Pacific Time) · Fax +1 3606471445

SPIE.org

and

The Optical Society

2010 Massachusetts Ave., N.W., Washington, D.C., 20036 USA

Telephone +1 2022238130 (Eastern Time) · Fax +1 2022231096

osa.org

Copyright (C) 2019, Society of Photo-Optical Instrumentation Engineers and The Optical Society

Copying of material in this book for internal or personal use, or for the internal or personal use of specific clients, beyond the fair use provisions granted by the U.S. Copyright Law is authorized by SPIE subject to payment of copying fees. The Transactional Reporting Service base fee for this volume is $\$ 21.00$ per article (or portion thereof), which should be paid directly to the Copyright Clearance Center (CCC), 222 Rosewood Drive, Danvers, MA 01923. Payment may also be made electronically through CCC Online at copyright.com. Other copying for republication, resale, advertising or promotion, or any form of systematic or multiple reproduction of any material in this book is prohibited except with permission in writing from the publisher. The CCC fee code is 1605 $7422 / 19 / \$ 21.00$.

Printed in the United States of America by Curran Associates, Inc., under license from SPIE.

Publication of record for individual papers is online in the SPIE Digital Library.

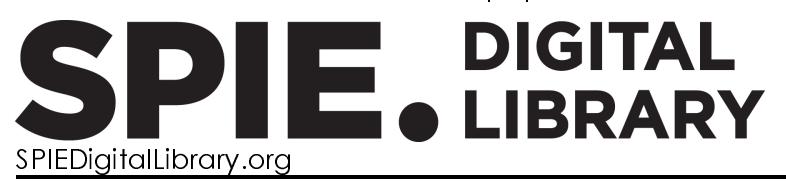

Paper Numbering: Proceedings of SPIE follow an e-First publication model. A unique citation identifier (CID) number is assigned to each article at the time of publication. Utilization of CIDs allows articles to be fully citable as soon as they are published online, and connects the same identifier to all online and print versions of the publication. SPIE uses a seven-digit CID article numbering system structured as follows:

- The first five digits correspond to the SPIE volume number.

- The last two digits indicate publication order within the volume using a Base 36 numbering system employing both numerals and letters. These two-number sets start with 00, 01, 02, 03, 04, 05, 06, 07, 08, 09, 0A, OB ... 0Z, followed by 10-1Z, 20-2Z, etc. The CID Number appears on each page of the manuscript. 


\title{
Contents
}

\author{
ix Authors \\ xiii Conference Committee
}

\section{SESSION 1 CLINICAL APPLICATIONS: SPECTROSCOPY}

1107302 Identification of amyloid-beta (A $\beta$ ) plaques in freshly frozen human brain tissue using Raman spectroscopy (Invited Paper) [1 1073-1]

1107304 Infrared spectroscopy evaluation of burn wound healing: semi-quantitative study [1 1073-3]

1107305 Label-free spectroscopic diagnosis of urothelial carcinoma [1 1073-4]

1107306 Raman spectroscopic investigation of plasma by drop coating deposition for clinical application [1 1073-5]

\section{SESSION 2 CLINICAL APPLICATIONS: IMAGING}

$1107307 \quad$ Design of a multi-modality salpingoscope for visualization of the ovary and oviducts (Invited Paper) [1 1073-6]

1107309 Real time intraoperative functional brain mapping using a RGB camera [1 1073-8]

$110730 \mathrm{~A}$ Cervical cancer diagnostics with a multispectral Mueller polarimetric colposcope [1 1073-9]

11073 OB Study of skin cancer lesions through multispectral and 3D techniques [1 1073-10]

SESSION $3 \quad$ NOVEL TECHNOLOGIES IN OPTICAL DIAGNOSTICS I

11073 OC In vivo testing of a CMOS-based diffuse reflectance device for skin condition monitoring (Invited Paper) [1 1073-11]

11073 OD Remote photoplethysmography for skin perfusion monitoring using narrowband illumination [11073-12]

11073 OE Feasibility of supercontinuum sources for use in glucose sensing by absorption spectroscopy [11073-13]

11073 OF Mueller polarimetric imaging through a rigid endoscope [1 1073-14] 
11073 OG Improving depth sensitive fluorescence spectroscopy with wavefront shaping [1 1073-15]

$11073 \mathrm{OH} \quad$ Ultra-miniature (diameter: $6 \mathrm{~mm}$, thickness: $5 \mathrm{~mm}$ ) low-cost (price: 1,000 EUR) point-one-shot mid-infrared Fourier spectroscopic imager for ear clip type non-invasive blood glucose sensors [1 1073-16]

SESSION 4 NOVEL METHODS FOR CELL AND TISSUE ANALYSIS

11073 0J Doppler imaging of intracellular dynamics in clinical cancer chemotherapy (Invited Paper) [1 1073-18]

11073 OK 3D large-volume histological imaging of ex vivo tissue samples using inverted selective plane illumination microscopy (iSPIM) [1 1073-19]

$11073 \mathrm{OL} \quad$ Monitoring temperature induced phase changes in subcutaneous fatty tissue using an astigmatism corrected dynamic needle probe [1 1073-20]

11073 OM Cell trauma detection using infra-red live cell imaging [1 1073-21]

11073 ON 850-nm near-infrared-ray computed tomography with high spatial resolutions [1 1073-22]

SESSION 5 TISSUE CHARACTERIZATION AND ANALYSIS

1107300 Determination of optical properties of human tissues obtained from parotidectomy in the spectral range of 250 to $800 \mathrm{~nm}$ [11073-23]

11073 OP Identification of azimuthal light scattering signatures to selectively track changes in subnuclear refractive index profile of epithelial cell models [1 1073-24]

11073 OR Study of malignant brain gliomas using optical coherence tomography and terahertz pulsed spectroscopy aimed on advanced intraoperative neurodiagnosis [1 1073-26]

11073 OS Assessing the spectrochemical signatures of skin components using FTIR microspectroscopy [1 1073-27]

11073 OT 5-ALA induced PpIX fluorescence guided surgery: a clinical study of spectral complexity in healthy tissues and margin boundaries in high and low grade gliomas [1 1073-28]

SESSION $6 \quad$ NOVEL TECHNOLOGIES IN OPTICAL DIAGNOSTICS II

11073 OU An endomicroscopic OCT for clinical trials in the field of ENT (Invited Paper) [1 1073-29]

11073 OV Real-time multispectral optical imaging using GPGPU processing [1 1073-30]

iv 
11073 OW Handheld OCT probe for intraoral diagnosis on teeth [1 1073-31]

11073 OX Rapid intraoperative margin assessment by using multi-modal third-harmonic generation and three-photon fluorescence microscopy [1 1073-32]

11073 OY Hyperspectral eye fundus imaging with extended spectral range towards the near infrared [1 1073-33]

\section{SESSION 7 NOVEL TECHNOLOGIES FOR IN VITRO DIAGNOSTICS}

1107310 Raman micro-spectroscopy investigation on the effects of $x$-rays and polyphenols in human neuroblastoma cells [1 1073-35]

$1107311 \quad$ Automated readout of a SERS lateral flow assay [11073-36]

1107312 Drop-coating deposition surface-enhanced Raman spectroscopy on silver substrates for biofluid analysis [1 1073-37]

$1107313 \quad$ Improved forward scatter detection of a flow cytometer for detection of extracellular vesicles [1 1073-38]

1107314 A compact multichannel spectrometer for label-free monitoring of biochips for point-of-care testing [1 1073-39]

1107315 Potential of bacterial infection diagnosis using infrared spectroscopy of WBC and machine learning algorithms [1 1073-40]

\section{SESSION 8 COMPUTATIONAL ANALYSIS AND MACHINE LEARNING}

1107316 Assessment of sampling adequacy using persistent homology for the evaluation of heterogeneity in 3D histology acquired through inverted selective plane illumination microscopy (iSPIM) (Invited Paper) [1 1073-41]

$1107317 \quad$ Pixel-wise modified Beer-Lambert model for intraoperative functional brain mapping [1 1073-42]

1107318 Objective and quantitative analysis of corneal transparency with clinical spectral-domain optical coherence tomography [1 1073-43]

1107319 Analysis of in vivo optical coherence tomography images of human peripheral nerves using texture analysis [1 1073-44]

$110731 \mathrm{~A} \quad$ Classification and identification of human colon cancer cell line in terahertz domain using tSNE [1 1073-45] 
11073 1B Longitudinal monitoring of in-vivo mice mammary tumor progression using intravital fluorescence tomography and optical coherence tomography (Invited Paper) [1 1073-46]

11073 1C Depth sensitive Raman spectroscopy for skin wounds in rodents [1 1073-47]

11073 ID Label-free fumor detection with active infrared thermal laparoscopic system in a mouse tumor model [1 1073-48]

$110731 \mathrm{E} \quad$ In vivo optical coherence tomography of a mouse model of spontaneous ovarian cancer [1 1073-49]

11073 1F Remote photoplethysmography for assessment of oral mucosa [1 1073-50]

$110731 \mathrm{G}$ Discrimination of brain tumours and dysplastic tissues through multimodal fibre-probe spectroscopy [1 1073-51]

$110731 \mathrm{H} \quad$ Colorectal polyps mimicking phantoms for OCT application [1 1073-52]

\section{POSTER SESSION}

$1107311 \quad$ Non-invasive LED-based screening solution for skin cancer [1 1073-17]

$110731 \mathrm{~J} \quad$ Optical coherence tomography (OCT) imaging of chronic lung allograft dysfunction (CLAD) [1 1073-53]

$110731 \mathrm{~K} \quad$ Endoscopic optical coherence tomography at the middle ear diagnostic [1 1073-54]

$110731 \mathrm{M} \quad \mathrm{X}$-ray irradiation effects on SH-SY5Y human neuroblastoma cells monitored by means of FTIR micro-spectroscopy [1 1073-56]

$110731 \mathrm{~N} \quad$ In vivo multimodal fibre-probe spectroscopy for glioblastoma detection in mouse model [1 1073-57]

11073 IP Diagnosis of inflammatory diseases of the paranasal sinuses using digital diaphanoscopy [1 1073-59]

11073 1R Blood plasma separation using microfluidic guiding channel in a continuous fashion [11073-61]

11073 is Imaging of LED-excited autofluorescence photobleaching rates for skin lesion diagnostics [1 1073-63]

$110731 \mathrm{~T} \quad$ Fluorescence from human oral cavity and body fluid saliva for detection of oral precancer: a comparison [1 1073-64]

11073 IV Monitoring x-rays exposed and unexposed cell culture media by means of surface-enhanced Raman spectroscopy [1 1073-66] 
11073 IW Fluorescent imaging of polystyrene microspheres with cyanine dyes for immunofluorescence analysis [1 1073-67]

$110731 \mathrm{X}$ Terahertz time-domain spectroscopy for human gastric cancer diagnosis [1 1073-68]

$11073 \mathrm{IY} \quad$ Breath analysis using cavity ring down spectroscopy in UV region for diagnostics of diabetes [1 1073-70]

$110731 \mathrm{Z} \quad$ Lymphedema tissue analysis using optical imaging and gradient processing [11073-71]

$1107321 \quad$ Measurement of human-body-window spectra using a white power light-emitting diode and its application to high-spatial-resolution computed tomography [1 1073-74]

$1107322 \quad$ Modulation interference microscopy as a promising method for assessing the risk of metastasis in patients with breast cancer [1 1073-75] 
Proc. of SPIE Vol. 11073 1107301-8

\section{Downloaded From: https://www.spiedigitallibrary.org/conference-proceedings-of-spie on 26 Apr 2023
Terms of Use: https://www.spiedigitallibrary.org/terms-of-use}




\section{Authors}

Numbers in the index correspond to the last two digits of the seven-digit citation identifier (CID) article numbering system used in Proceedings of SPIE. The first five digits reflect the volume number. Base 36 numbering is employed for the last two digits and indicates the order of articles within the volume. Numbers start with 00, 01, 02, 03, 04, 05, 06, 07, 08, 09, OA, OB...0Z, followed by 10-12, 20-2Z, etc.

Abola, A., 1Y

Adachi, Satoru, $\mathrm{OH}$

Agbaria, Adam H., 15

Aglinska, A., OD

Aguenounon, Fabrice, OC, OV

Ahrens, M., OU, OW

Aksnes, Astrid, OE

Aleksandrova, Polina $\vee$., OR

Ali, Karimi, 1R

Alston, L., OT

Alterini, T., OY

Anand, Suresh, 05, 1G

Arens, Philipp, 00

Ares, Miguel, OB

Ariese, Freek, 02

Arifler, Dizem, $\mathrm{OP}$

Artyushenko, V. G., 1P

Balkanov, Andrey, 22

Baria, Enrico, 05, 1G, 1N

Baritaux, Jean-Charles, 11

Barman, Ishan, 12

Barton, Jennifer K., 07, $1 \mathrm{E}$

Batjargal, Orkhongua, 07

Beck, Guy, 15

Becker, David L., 1C

Belolipetskaya, Julia, 1X

Bernier, Damien, 14

Beshplav, Sheykh-Islyam T., OR

Bibikova, O. A., IP

Birngruber, R., OL

Bliznuks, Dmitrijs, 1S

Bloemen, P. R., 13

Bocheux, Romain, 18

Bocklitz, Thomas, 06

Bondina, Ekaterina V., IW

Borderie, Vincent, 18

Borisov, Alexey V., 12

Bornitz, Matthias, $1 \mathrm{~K}$

Brenke, C., 19

Brown, J. Quincy, OK, 16

Bryanskaya, E. O., 1 P

Buccoliero, Anna Maria, $1 G$

Bukin, A. G., 1 P

Burgos-Fernández, Francisco J., OB

Byrne, Hugh J., OS

Caica, A., OD

Camerlingo, Carlo, $1 \mathrm{~V}$

Cao, Yuqi, $1 \mathrm{~A}$

Capmas, Perrine, $\mathrm{OA}$
Capozzi, Vito, 10

Caravaca, Oscar, $1 \mathrm{H}$

Caredda, Charly, 09, 17

Carolus, A., 19

Casper, M., OL

Casquel, Rafael, 14

Chaible, Lucas M., 1B

Chaker, A., OU

Chen, Jiani, $1 \mathrm{~A}$

Chernomyrdin, Nikita $\mathrm{V}$., OR

Chun, Honggu, 1R

Chung, Euiheon, 1D

Ciaurriz, Paula, 14

Cicchi, Riccardo, 05, 1G, 1N

Cornago, Iñaki, 14

Correa, Luciana, OS

Coumans, F. A. W., 13

Dadouche, Foudil, OV

de Boer, Johannes F., 02

Debras, Elodie, OA

de Castro, Pedro A. A., 04

Dehghani, Hamid, OM

Delfino, Ines, 10, $1 \mathrm{~V}$

Delpueyo, Xana, OB

Demchenko, P.S., $1 \mathrm{X}$

Denti, Maxime, 14

Deprez, Sylvain, 14

Derjabo, Aleksandrs, 11, 1S

de Rond, L., 13

Díaz-Doutón, F., OY

Dinten, Jean-Marc, OC

Dolganova, Irina N., OR

Dolgushin, Sergey A., IW

Dommerich, Steffen, 00

Dortu, Fabian, 14

Dunaev, A. V., 1 P

Eisert, Peter, 00

Ellingsen, Reinold, OE

Enomoto, Toshiyuki, 21

Evers, M., OL

Fantechi, Riccardo, 05

Fasy, Brittany Terese, 16

Fernandez, Fátima, 14

Fernandez, Hervé, OA

Fesus, Luca, 11

Fuglerud, Silje S., OE

Gacci, Mauro, 05

Galvez, Dominique B., 07

Gavdush, Arsenii A., OR 
Ge, Weiting, 1A

Gennet, Camille, OA, OF

Georgeon, Cristina, 18

Gerbelot, Rémi, OC

Gerhardt, N. C., 19

Giordano, Flavio, $1 \mathrm{G}$

Gioux, Sylvain, OC, OV

Golde, Jonas, $1 \mathrm{~K}$

Gora, Michalina, 1H

Grabovskis, A., OD, $1 \mathrm{~F}$

Grateau, Henri, OC

Gravelyn, Sara, $1 \mathrm{H}$

Grigorev, Roman, $1 \mathrm{X}$

Grundsteins, K., $1 Y$

Guillaud, Martial, OP

Guo, Shuxia, 06

Guyotat, Jacques, 09, 0T, 17

Haak, R., OW

Häfer, M., OW

Hagiwara, Osahiko, 21

Hastanin, Jurij, 14

Hebert, M., OT

Henkuzena, I., $1 \mathrm{~F}$

Hernandez, Ana L., 14

Hernandez, Yves, 14

Hilsmann, Anna, 00

Hjelme, Dag R., OE

Hoang, Antoine, 11

Hofmann, M. R., 19

Hohert, Geoffrey, 1J

Holgado, Miguel, 14

Hoozemans, Jeroen J. M., 02

Hou, Dibo, 1A

Howle, Christopher R., OM

Hsieh, Chao-Mao, OG

$\mathrm{Hu}$, Bihe, OK, 16

Huang, Jing, 06

Huang, Pingjie, 1A

Huang, Zufang, 12

Huleihel, Mahmoud, 15

Hüttmann, G., OL, OU, OW

Idel, C., OU

Ignatiev, Pavel, 22

Irsch, Kristina, 18

Ishimaru, Ichiro, $\mathrm{OH}$

Jalal, Shadia, OJ

Jallon, Pierre, OC

Jechlinger, Martin, 1B

Jung, Gyeong Bok, $1 \mathrm{R}$

Kang, Hanyue, $\mathrm{OH}$

Kang, Yang Jun, $1 \mathrm{R}$

Kantapareddy, P., OT

Kao, Chien-Ting, ox

Kapelushnik, Joseph, 15

Kawashima, Natsumi, $\mathrm{OH}$

Kemper, Max, 1K

Khamid, Abdo, 1X

Khodzitsky, Mikhail, $1 \mathrm{X}$

Kiehntopf, Michael, 06

Kiekens, Kelli C., 07
Kieu, Khanh, 07

Kirsten, Lars, 1K

Kiss, Norbert, 11

Kistenev, Yury V., 12

Kitazaki, Tomoya, $\mathrm{OH}$

Knyazkova, Anastasya I., 1 Z

Koch, Edmund, 1K

Koenig, Anne, OC

Koevary, Jennifer W., $1 \mathrm{E}$

König, P., OU

Kosyrkova, Alexandra V., OR

Kryvova, Natalya A., 1 Z

Kumar, Pavan, $1 \mathrm{~T}$

Kurasova, Anna, 1X

Kuzikova, Anna, $1 \mathrm{X}$

Lane, Pierre, $1 \mathrm{~J}$

Lange, Marta, 11, 1S

Lapidot, Itshak, 15

Lasalvia, Maria, 10

Lawson, Peter, 16

Lee, Anthony M. D., $1 \mathrm{~J}$

Lenaerts, Cédric, 14

Lenz, M., 19

Lepore, Maria, 10, 1M, 1V

Li, Guang, OK

Liao, Yi-Hua, OX

Lihachev, Alexey, 1S

Lihacova, llze, $1 \mathrm{~s}$

Lima, Cassio A., OS

Lindberg, Arvid, OA, OF

Liv, Quan, 0G, $1 C$

Lochocki, Benjamin, 02

Madden, Leigh, $1 \mathrm{C}$

Mahieu-Williame, Laurent, 09, 0T, 17

Maigler, María V., 14

Makovik, I. N., IP

Malone, Jeanie, 1J

Malvehy, Josep, OB

Manstein, D., OL

Manti, Lorenzo, 10, 1M

Marcinkevics, Z., OD, $1 \mathrm{~F}$

Maricot, Sophie, 14

Matei, Daniela E., OJ

Mellors, Ben O. L., OM

Metelin, Vladislav, 22

Meyer-Schell, N., OL

Meyronet, D., OT

Miemiec, R., 19

Milenko, Karolina, OE

Minet, O., 1P

Möller, J., 19

Montcel, Bruno, 09, 0T, 17

Montero, Marta G., 1B

Mordechai, Shaul, 15

Morel, Nathalie, 11

Morgenstern, Joseph, 1K

Moriyama, Hodaka, ON, 21

Morrema, Tjado H. J., 02

Morselli, Simone, 05

Muckle, R. A., $1 \mathrm{~F}$ 
Nador, Roland, $1 \mathrm{~J}$

Nazac, André, OA

Neudert, Marcus, $1 \mathrm{~K}$

Neugebauer, Ute, 06

Nikitin, Pavel V., OR

Nikolaev, Viktor V., 1 Z

Nishiyama, Akira, $\mathrm{OH}$

Nolte, David D., OJ

Noutsias, Michel, 06

Oda, Yasuyuki, ON, 21

Oh, Gyungseok, 1D

$\mathrm{Ou}, \mathrm{Yi}-\mathrm{H} \sin , \mathrm{O}$

Ozturk, Mehmet S., 1B

Pacifico, Severina, 10

Park, Junha, OA

Parkhomchuk, Ekaterina V., IW

Pavone, Francesco Saverio, 05, 1G, iN

Pellacani, Giovanni, OB

Perna, Giuseppe, 10

Pernot, Pascal, 18

Petitdidier, Nils, OC

Piccolella, Simona, 10

Pierangelo, Angelo, OA, OF

Pillai, Vinoshene, $1 \mathrm{~N}$

Plamann, Karsten, 18

Plorina, Emilija Vija, 11, 1S

Popp, Jürgen, 06

Portaccio, Marianna, $1 \mathrm{M}$

Pracucci, Enrico, $1 \mathrm{~N}$

Pradhan, Asima, $1 T$

Prevedel, Robert, 1B

Puig, Susana, OB

Ramoji, Anuradha, 06

Ratto, Gian Michele, $1 \mathrm{~N}$

Rehbinder, Jean, OA

Reshetov, Igor $V$., OR

Revalde, G., IY

Rey-Barroso, Laura, OB

Ricciardi, Valerio, 10, $1 \mathrm{M}$

Rice, Photini F. S., $1 \mathrm{E}$

Rich, Daniel H., 15

Rivière, Bathilde, 18

Romano, Gabriella, 07

Roobroek, Aline, 14

Rousseau, D., OT

Royo, Santiago, OB

Roze, A., $1 \mathrm{~F}$

Rubins, U., OD, $1 \mathrm{~F}$

Rüger, C., OW

Sablong, Raphaël, 09, 17

Salman, Ahmad, 15

Sandykova, Ekaterina A., 12

Sankova, Natalya N., 1W

Sato, Eiichi, ON, 21

Sato, Yuichi, ON

Sawyer, Travis W., $1 \mathrm{E}$

Schindler, Martin, $1 \mathrm{~K}$

Schmieder, K., 19

Schneider, H., OW

Schulz-Hildebrandt, H., OL, OU, OW
Sdika, Michaël, 09, 17

Sebastianelli, A., 05

Semeykina, Victoria S., IW

Senyuk, Artem, $1 \mathrm{X}$

Serni, Sergio, 05

Shalaev, Pavel V., IW

Shuraev, B. M., IP

Siddhanta, Soumik, 12

Spear, Abigail M., OM

Spigulis, Janis, 11

Strumpski, M., OW

Su, Joshua W., $1 C$

Summa, Brian, 16

Sun, Chi-Kuang, OX

Tellechea, Edurne, 14

Teo, Erica M. L., 1C

Theisen-Kunde, D., OW

Thurgood, Harrison T., 07

Tian, Yao, $1 \mathrm{C}$

Tuchin, Valery $\mathrm{V}$., OR

Turek, John J., OJ

Uecker, Florian C., 00

Uhring, Wilfried, OV

van der Pol, E., 13

Vanel, Jean-Charles, OA, OF

van Leeuwen, T. G., 13

Vasilenko, Irina, 22

Veenstra, Theo, 14

Vega, David, 07

Vilaseca, Meritxell, OB, OY

Vilcot, Jean-Pierre, 14

Vizet, Jérémy, OA, OF

Von Horsten, Hendrik, 14

Vrazhnov, Denis A., 1 Z

Wada, Kenji, $\mathrm{OH}$

Walther, Julia, $1 \mathrm{~K}$

Wang, Ling, 1B

Wang, Qiang, 1C

Watanabe, Manabu, ON, 21

Wei, Ming-Liang, OX

Welp, H., 19

Wenk, Carola, 16

Wisotzky, Eric L., 00

Wollenberg, B., OU

Wróbel, Maciej S., 12

Yoshida, Sohei, ON, 21

Zabarylo, U., 1P

Zahnert, Thomas, $1 \mathrm{~K}$

Zakharenko, Alexander, $1 \mathrm{X}$

Zaytsev, Kirill I., OR

Zezell, Denise M., 04, OS

Zhang, Guangxin, 1A

Zorn, Lucile, $1 \mathrm{H}$

Zulina, Natalia, $1 \mathrm{H}$ 
Proc. of SPIE Vol. 11073 1107301-12

Downloaded From: https://www.spiedigitallibrary.org/conference-proceedings-of-spie on 26 Apr 2023 Terms of Use: https://www.spiedigitallibrary.org/terms-of-use 


\title{
Conference Committee
}

\author{
General Chairs \\ Brett E. Bouma, Wellman Center for Photomedicine (United States) \\ Paola Taroni, Politecnico di Milano (Italy)
}

Programme Chairs

Ronald Sroka, Laser-Forschungslabor (Germany)

I. Alex Vitkin, University of Toronto (Canada)

Conference Chairs

J. Quincy Brown, Tulane University (United States)

Ton G. van Leeuwen, Academisch UMC (Netherlands)

Conference Programme Committee

Caroline Boudoux, Ecole Polytechnique de Montréal (Canada)

Daniel Cote, University Laval (Canada)

Kishan Dholakia, University of St. Andrews (United Kingdom)

Daniel S. Elson, Imperial College London (United Kingdom)

Sylvain Gioux, Université de Strasbourg (France)

Jonathan T. C. Liu, University of Washington (United States)

Quan Liu, Nanyang Technological University (Singapore)

Narasimhan Rajaram, University of Arkansas (United States)

Lise Lyngsnes Randeberg, Norwegian University of Science and

Technology (Norway)

Daniel Razansky, Helmholtz Zentrum München GmbH (Germany)

Darren M. Roblyer, Boston University (United States)

Göran Salerud, Linköping University (Sweden)

Janis Spigulis, University of Latvia (Latvia)

Henricus J. C. M. Sterenborg, Academisch Medisch Centrum

(Netherlands)

James W. Tunnell, The University of Texas at Austin (United States)

Karthik Vishwanath, Miami University (United States)

Siavash Yazdanfar, Corning Incorporated (United States)

\section{Session Chairs}

1 Clinical Applications: Spectroscopy

Jonathon Quincy Brown, Tulane University (United States)

Ton G. van Leeuwen, Amsterdam UMC (Netherlands) 
2 Clinical Applications: Imaging

Sylvain Gioux, Laboratoire des sciences de l'ingénieur, de l'informatique et de l'imagerie (France)

3 Novel Technologies in Optical Diagnostics I

Kishan Dholakia, University of St. Andrews (United Kingdom)

$4 \quad$ Novel Methods for Cell and Tissue Analysis

J. Quincy Brown, Tulane University (United States)

5 Tissue Characterization and Analysis

Sylvain Gioux, Laboratoire des sciences de l'ingénieur, de l'informatique et de l'imagerie (France)

6 Novel Technologies in Optical Diagnostics II

Siavash Yazdanfar, Corning Incorporated (United States)

$7 \quad$ Novel Technologies for In Vitro Diagnostics

Janis Spigulis, University of Latvia (Latvia)

8 Computational Analysis and Machine Learning

Ton G. van Leeuwen, Amsterdam UMC (Netherlands)

9 In Vivo Imaging and Spectroscopy

Jonathon Quincy Brown, Tulane University (United States)

Ton G. van Leeuwen, Amsterdam UMC (Netherlands) 